\title{
Real-time Reactive Trip Avoidance for Powered Transfemoral Prostheses
}

\author{
Nitish Thatte*, Nandagopal Srinivasan ${ }^{\dagger}$, and Hartmut Geyer* \\ ${ }^{*}$ Robotics Institute, ${ }^{\dagger}$ Mechanical Engineering \\ Carnegie Mellon University, Pittsburgh, Pennsylvania 15213 \\ Email: nitisht@cs.cmu.edu, nandagos@andrew.cmu.edu, hgeyer@cs.cmu.edu
}

\begin{abstract}
This paper presents a real-time reactive controller for a powered prosthesis that addresses the problem of trip avoidance. The control estimates the pose of the leg during swing with an extended Kalman filter, predicts future hip angles and hip heights using sparse Gaussian Processes, and reactively plans updated ankle and knee trajectories with a fast quadratic program solver to avoid trips. In preliminary experiments with an able-bodied user who purposefully lowered the hip to elicit trips on each swing, the proposed control reduced the rate of tripping by $68 \%$ when compared to a swing control that follows standard minimumjerk trajectories. In addition, the proposed control also reduced the severity of toe-scuffing. To the best of our knowledge, this controller is the first to incorporate visual feedback in the realtime planning and control of a lower limb prosthesis during gait. The results demonstrate the potential of reactive and environmentaware controls to improve amputee gait robustness and encourage future development of leg prosthesis controls that can react in real-time to the environment and user state.
\end{abstract}

\section{INTRODUCTION}

Lower limb amputees using state-of-the-art commercial prostheses face a number of gait deficiencies that negatively impact their quality of life [6]. Of acute significance among these deficiencies are the increased risk of falling and the related injuries, which can lead to amputees avoiding activity out of a fear falling [16]. As falls and their avoidance are linked to swing leg placement in locomotion, active swing control strategies could help to substantially reduce the risk of falling. However, current swing controllers of transfemoral prostheses do little to proactively minimize this risk.

Existing swing phase control approaches for powered prostheses predominantly seek to reproduce the average swing phase behavior of the human leg. Whether the approach is based on trajectory planning [12], impedance control [24], or phasebased control [18], they all treat the swing phase motion as an "open loop" problem with respect to trip hazards, as none of the approaches take the location of the heel and toe of the prosthetic foot with respect to the ground explicitly into account. Therefore, current swing control strategies neglect a clear advantage that robotic prostheses can have over their passive counterparts: the ability to sense and act upon environmental information.

In this work, we develop a swing control strategy to reactively avoid trips with powered transfemoral prostheses that uses visual information about the environment and an estimate of the prosthesis configuration. Some previous studies have explored incorporating visual feedback into the control of leg prostheses. For example, Scandaroli et al. [21] developed a state estimator and controller that allowed the ankle joint of a prosthesis to conform to the slope of the ground under the foot. To address the problem of terrain recognition, Zhang et al. [27] developed a classifier using a LIDAR and an IMU to discriminate between terrains such as flat ground and steps. More recently, Liu et al. [13] combined this terrain classifier with a Bayesian intent classifier (based on [3]) to develop an environment-aware locomotion mode recognition system. In addition, RGBD sensors have been explored as a source of rich environmental information for legged assistance, including gait recognition [15] and stair detection $[4,10]$. However, none of these previous studies have implemented a control strategy that uses information about the state of the prosthesis with respect to its environment to reactively govern the motion of a powered prosthesis in real-time. Hayashi and Kiguchi [8] do propose such a control strategy for a powered exoskeleton that assists with obstacle avoidance. This work used a terrain map generated by a scanning LIDAR and sensors on both the stance and swing legs to provide assistive torques that prevent trips.

Here, we present an approach for real-time, reactive tripavoidance control of a powered prosthesis that does not require stance leg sensing and uses a simple 1-D LIDAR distance sensor. The approach combines three building blocks. First, we use an extended Kalman filter (EKF) that fuses measurements from an IMU, a 1-D LIDAR, and the prosthesis' encoders to estimate the current pose of the prosthetic leg with respect to the ground. Second, during swing we predict likely future leg trajectories with sparse Gaussian process models. Finally, we use the leg pose estimate and trajectory predictions in a fast quadratic-program planner to reactively generate in real time leg joint trajectories that avoid premature toe and heel contact with the ground. To evaluate the proposed control, we compare our method for trip avoidance to a standard non-reactive minimum-jerk trajectory planning approach in a prosthesis walking experiment designed to elicit trips.

\section{Methods}

The trip avoidance control we propose involves (1) estimating the position and orientation of the leg (section II-A), (2) predicting the future hip angles and heights (section II-B), and (3) planning corresponding knee and ankle trajectories such that the heel and toe will not contact the ground prematurely (section II-C). 


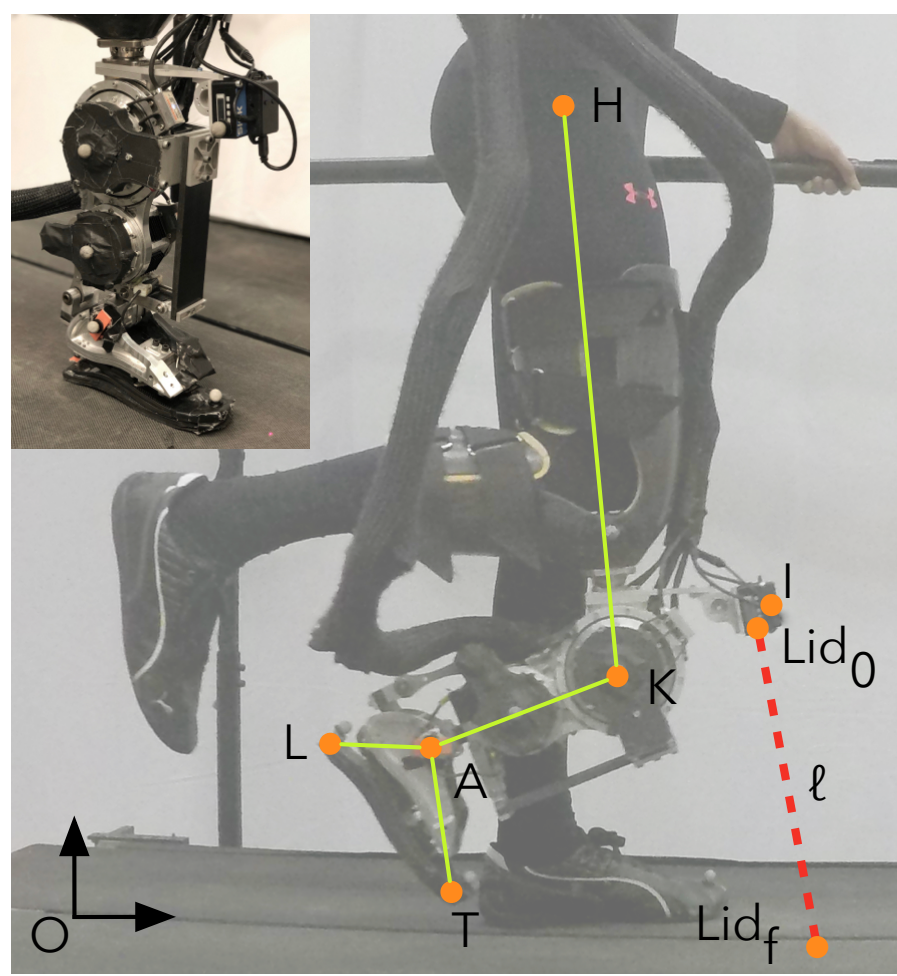

Fig. 1: Kinematic model of the user and prosthesis used for state estimation and motion planning. The model includes the hip $(\mathrm{H})$, knee $(\mathrm{K})$, ankle (A), heel (L) and toe points (T). Additionally, the start $\left(\operatorname{Lid}_{0}\right)$ and end $\left(\operatorname{Lid}_{f}\right)$ points of the LIDAR beam (with length $\ell$ ) are indicated. The IMU is located at point $I$. Both the LIDAR and IMU are mounted to the thigh portion of the powered knee-and-ankle prosthesis.

\section{A. Extended Kalman Filter for estimating Leg Position/Orientation}

To estimate the position and orientation of the leg, we employ an EKF that fuses information from a LIDAR distance sensor (SICK OD1000), an IMU (YEI Technologies 3-Space sensor), and encoders on the prosthesis (Renishaw Resolute, Netzer DS25). The EKF filters the nonlinear, discrete-time dynamics given by

$$
\begin{aligned}
& x_{t}=\left[\begin{array}{c}
q_{t} \\
p_{t} \\
\dot{p}_{t}
\end{array}\right]=\left[\begin{array}{ccc}
f_{\mathrm{gyro}}\left(\omega_{t}\right) & 0 & 0 \\
0 & I_{3 \times 3} & \Delta t I_{3 \times 3} \\
0 & 0 & I_{3 \times 3}
\end{array}\right] x_{t-1} \\
& +\left[\begin{array}{c}
0 \\
\frac{1}{2} \Delta t^{2} I_{3 \times 3} \\
\Delta t I_{3 \times 3}
\end{array}\right]\left[R_{\mathrm{OI}}\left(q_{t-1}\right) a_{t}-\left[\begin{array}{l}
0 \\
0 \\
g
\end{array}\right]\right]+w_{t} \\
& =f\left(x_{t-1}, u_{t}\right)+w_{t} \text {, }
\end{aligned}
$$

where $q$ is the quaternion orientation, $R_{\mathrm{OI}}$ and $p$ are the rotation matrix and position of the IMU in inertial coordinates, $\omega$ is the bias-corrected angular rate measured by the gyroscope, $f_{\text {gyro }}$ integrates the gyroscope rate to update the orientation (for more details see [14]), $a$ is the accelerometer measurement, $u_{t}=\left[\omega_{t}, a_{t}\right]^{T}$, and $\Delta t$ is the integration time step (1 ms).
The dynamics are corrupted by process noise $w_{t} \sim \mathcal{N}\left(0, Q_{t}\right)$ due to the inaccuracy of the IMU's measurement of the true acceleration and angular velocity. Consequently, $Q_{t}$ is given by

$$
Q_{t}=\left.\left.\frac{\partial f}{\partial u}\right|_{x_{t-1}, u_{t}}\left[\begin{array}{cc}
\sigma_{\omega}^{2} I_{3 \times 3} & 0 \\
0 & \sigma_{a}^{2} I_{3 \times 3}
\end{array}\right] \frac{\partial f}{\partial u}\right|_{x_{t-1}, u_{t}} ^{T},
$$

where $\sigma_{\omega}^{2}$ and $\sigma_{a}^{2}$ are the gyroscope and accelerometer measurement variances, respectively.

To estimate the pose given our sensor measurements, we follow a standard EKF procedure [1], reviewed here for completeness. The EKF state estimation process has two steps: First, we predict the next state distribution by forward-propagating the mean $\hat{x}_{t-1 \mid t-1}$ and covariance of the state estimate $\Sigma_{t-1 \mid t-1}$ using the dynamics given by eq. (1),

$$
\begin{aligned}
\hat{x}_{t \mid t-1} & =f\left(\hat{x}_{t-1 \mid t-1}, u_{t}\right) \\
\Sigma_{t \mid t-1} & =F_{t} \Sigma_{t-1 \mid t-1} F_{t}^{T}+Q_{t},
\end{aligned}
$$

where $F_{t}=\partial f /\left.\partial x\right|_{\hat{x}_{t-1 \mid t-1}}$.

Next, we incorporate information from noisy sensor observations to update the state estimate. To do this, we utilize a observation model given by $z_{t}=h\left(x_{t}\right)+v_{t}$, where $v_{t} \sim \mathcal{N}(0, R)$, and the following update equations:

$$
\begin{aligned}
K_{t} & =\Sigma_{t \mid t-1} H_{t}^{T}\left(H_{t} \Sigma_{t \mid t-1} H_{t}^{T}+R\right)^{-1} \\
\hat{x}_{t \mid t} & =\hat{x}_{t \mid t-1}+K_{t}\left(z_{t}-h\left(\hat{x}_{t \mid t-1}\right)\right) \\
\Sigma_{t \mid t} & =\left(I-K_{t} H_{t}\right) \Sigma_{t \mid t-1}
\end{aligned}
$$

where $z_{t}$ are the actual sensor measurements and $H_{t}=$ $\partial h /\left.\partial x\right|_{\hat{x}_{t-1 \mid t}}$.

The observations in our EKF formulation use the kinematic model shown in fig. 1. We calibrate this model using ground truth data from a VICON motion capture system. In our application we incorporate three observations:

1) The expected measurement vector from the IMU's accelerometer is primarily due to gravity and thus points up in the global coordinate frame. As $R_{O I}$ represents the coordinate system rotation between the global frame $O$ and the IMU's frame $I$, we expect the measured acceleration $a_{\text {meas }}$ to align with the third row of $R_{O I}$,

$$
\begin{aligned}
h_{1}\left(x_{t}\right) & =\left\{R_{\mathrm{OI}}(q)\right\}_{\text {row } 3} \\
z_{1} & =a_{\text {meas }}
\end{aligned}
$$

2) The expected LIDAR measurement given the position of the IMU,

$$
\begin{aligned}
h_{2}\left(x_{t}\right) & =\left\{\ell:\left\{p_{\mathrm{OLID}_{\mathrm{f}}}\left(x_{t}, \ell\right)\right\}_{\text {row } 3}=0\right\} \\
z_{2} & =\ell_{\text {meas }},
\end{aligned}
$$

where $p_{\mathrm{OLID}_{\mathrm{f}}}$ is the location of the laser beam endpoint represented in the global coordinate system, $\ell=\left\|\overrightarrow{\mathrm{LID}_{0} \mathrm{LID}_{\mathrm{f}}}\right\|$ is the modeled laser beam length, and $\ell_{\text {meas }}$ is the actual measured LIDAR distance. 
3) During stance, the toe point coincides with the origin (active $200 \mathrm{~ms}$ after stance begins until toe-off)

$$
\begin{aligned}
h_{3}\left(x_{t}\right) & =p_{\mathrm{OT}}\left(x_{t}, \theta_{\mathrm{k}}, \theta_{\mathrm{a}}\right) \\
z_{3} & =\left[\begin{array}{lll}
0 & 0 & 0
\end{array}\right]^{T}
\end{aligned}
$$

where $p_{\text {OT }}$ describes the location of the toe in the inertial frame as a function of the EKF's state estimate $x_{t}$ and the knee $\theta_{\mathrm{k}}$ and ankle $\theta_{\mathrm{a}}$ angles, which are measured by the prosthesis' joint encoders. This observation is inspired by previous work on pedestrian tracking across multiple steps, in which a pseudomeasurement constrains the IMU velocity to zero during stance [5].

The measurement noise for these observations is given by

$$
R=\left[\begin{array}{cc}
\sigma_{a}^{2} I_{3 \times 3} & 0 \\
0 & \sigma_{l}^{2}
\end{array}\right]
$$

during swing and

$$
R=\left[\begin{array}{ccc}
\sigma_{a}^{2} I_{3 \times 3} & 0 & 0 \\
0 & \sigma_{\ell}^{2} & 0 \\
0 & 0 & \sigma_{f}^{2} I_{3 \times 3}
\end{array}\right]
$$

during stance. In these equations, $\sigma_{a}^{2}$ is the accelerometer variance, $\sigma_{\ell}^{2}$ is the LIDAR measurement variance, and $\sigma_{f}^{2}$ is the foot position variance.

To further improve the EKF's state estimate, we enforce a number of constraints using the methods provided by Gupta and Hauser [7]. Specifically, we enforce three equality constraints:

1) First, we require that the quaternion has unit norm

$$
1=q_{1}^{2}+q_{2}^{2}+q_{3}^{2}+q_{4}^{2}
$$

2) Second, we prevent the yaw component of the orientation $q$ from drifting. To do this, we convert the $q$ to ZYX Euler angles and enforce $\phi_{z}=0$,

$$
0=\operatorname{atan} 2\left(2\left(q_{1} q_{4}+q_{2} q_{3}\right), 1-2\left(q_{3}^{2}+q_{4}^{2}\right)\right) .
$$

3) Finally, during stance we further constrain the toe's $x$ and $y$-coordinates to 0 ,

$$
\left[\begin{array}{l}
0 \\
0
\end{array}\right]=\left\{p_{\mathrm{OT}}\left(x_{t}, \theta_{\mathrm{k}}, \theta_{\mathrm{a}}\right)\right\}_{\text {rows } 1 \text { and } 2} \text {. }
$$

In addition, we use inequality constraints to ensure the toe and heel do not penetrate the ground,

$$
\begin{aligned}
& 0 \leq\left\{p_{\mathrm{OT}}\left(x_{t}, \theta_{\mathrm{k}}, \theta_{\mathrm{a}}\right)\right\}_{\text {row } 3}, \\
& 0 \leq\left\{p_{\mathrm{OL}}\left(x_{t}, \theta_{k}, \theta_{a}\right)\right\}_{\text {row } 3} .
\end{aligned}
$$

We enforce these constraints by solving the following quadratic program after each update step,

$$
\hat{x}_{t \mid t}^{\mathrm{proj}}=\operatorname{argmin}_{x}\left(x-\hat{x}_{t \mid t}\right)^{T} \Sigma_{t \mid t}^{-1}\left(x-\hat{x}_{t \mid t}\right),
$$

such that

$$
\begin{gathered}
A_{\text {eq }} x=b_{\text {eq }}, \\
A_{\text {ineq }} x=b_{\text {ineq }},
\end{gathered}
$$

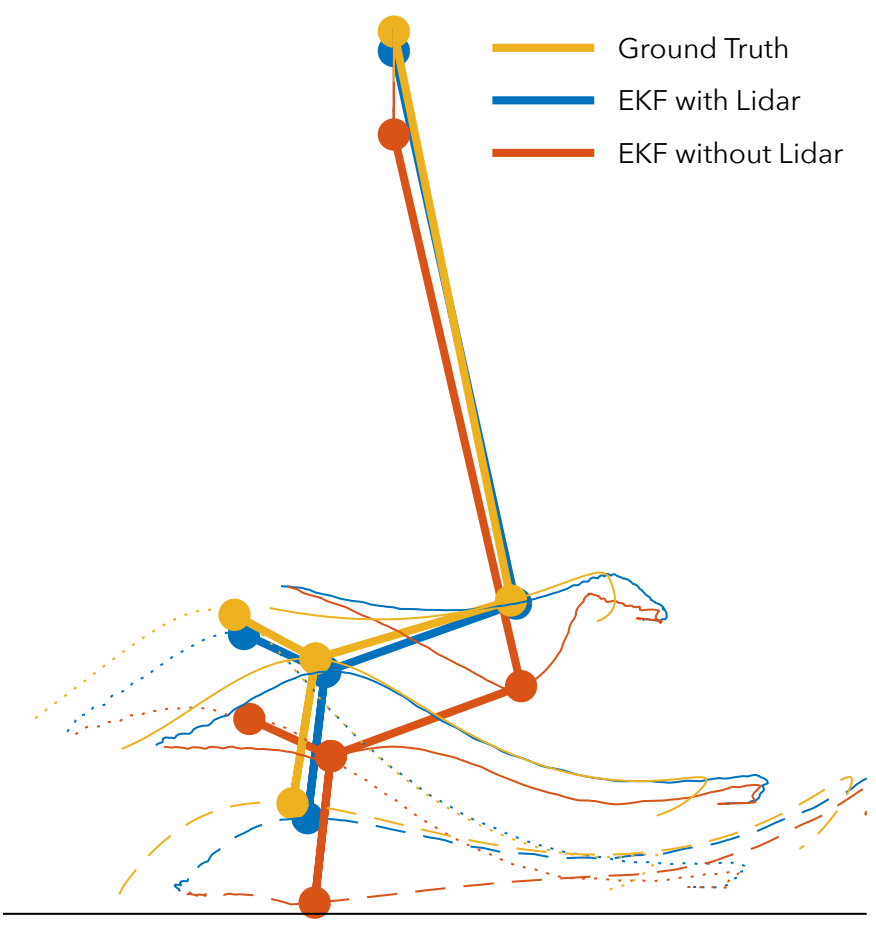

Fig. 2: Trajectories of extended Kalman Filter (EKF) estimate of the position of the leg during swing (blue). Ground truth positions given by motion capture (yellow). EKF estimate without LIDAR information shown in red. Thick lines show the leg configuration at peak toe height during swing. Dotted lines indicate heel trajectories while dashed lines show the toe trajectories. Knee and ankle trajectories given by solid lines.

where $A_{\text {eq }}, b_{\text {eq }}, A_{\text {ineq }}$, and $b_{\text {ineq }}$ are derived from linearizing the equality and inequality constraints. We found in practice that solving this $\mathrm{QP}$ a single time resulted in a state estimate that was sufficiently close to the constraints and that iteratively linearizing the constraints and solving the QP was unnecessary.

To identify the appropriate parameters for the EKF, we collected ground truth training and testing kinematic data using a Vicon motion capture system. We optimized the parameters of the EKF by simulating the state estimator with different parameters and evaluating the resulting error between the estimated trajectory and the ground truth trajectory. The parameters we optimized were the rotation of the LIDAR with respect to the hip, the translation between the LIDAR and the IMU, and $\sigma_{\omega}, \sigma_{a}, \sigma_{\ell}$, and $\sigma_{f}$.

Figure 2 shows an example of the resulting EKF estimates of the hip, knee, ankle, heel, and toe positions during swing (blue stick figure and traces) compared to the ground truth obtained from the motion capture system (yellow) and an EKF estimate without the LIDAR sensor information integrated (red). Over the entire test data set, the root mean squared error of the estimated heel and toe positions during swing is $18.6 \mathrm{~mm}$ for the EKF with LIDAR information. In contrast, the EKF without LIDAR information has an error of $46.7 \mathrm{~mm}$. Thus, including 
the LIDAR sensor data reduces the error by $60 \%$.

\section{B. Gaussian Process Hip Trajectory Prediction}

To predict the future hip trajectory, we learn Gaussian process models that describe the user's hip height and angle versus time during the swing phase. We train these models with data obtained from the specific user of the prosthesis during an initial data collection period in which a standard minimum-jerk approach generates the swing phase joint angle trajectories. We specifically train sparse Gaussian process models using the FITC approximation [23] which ensures the computational complexity at test time is independent of the training data set size, thereby providing consistent real-time performance. Throughout the swing phase, the learned hip angle and height distributions are conditioned on the swing trajectories completed so far to predict the distribution of the future trajectories for the rest of the swing (example shown in fig. 3). Our algorithm then uses the means of these conditional distributions in the motion planning phase (compare section II-C).

For example, to calculate the conditional mean of future hip angles, we first compute the joint distribution of completed $\left(\theta_{h}^{\mathrm{c}}\right)$ and future $\left(\theta_{h}^{\mathrm{f}}\right)$ hip angles,

$$
\begin{aligned}
\mathrm{P}\left(\theta_{h}^{\mathrm{c}}, \theta_{h}^{\mathrm{f}}\right) & =\mathcal{N}\left(\mu_{\text {fitc }}, \Sigma_{\text {fitc }}+K\left(t_{\text {joint }}, t_{\text {joint }}\right)\right) \\
& =\mathcal{N}\left(\left[\begin{array}{l}
\mu_{\mathrm{c}} \\
\mu_{\mathrm{f}}
\end{array}\right],\left[\begin{array}{cc}
\Sigma_{\mathrm{c}, \mathrm{c}} & \Sigma_{\mathrm{c}, \mathrm{f}} \\
\Sigma_{\mathrm{f}, \mathrm{c}} & \Sigma_{\mathrm{f}, \mathrm{f}}
\end{array}\right]\right),
\end{aligned}
$$

where $\mu_{\text {fitc }}$ and $\Sigma_{\text {fitc }}$ are obtained from equation 11 in [23] and $K\left(t_{\text {joint }}, t_{\text {joint }}\right)$ is an additional noise term given by a rational quadratic kernel [19] that correlates the predicted angles across time, which results in smooth predicted trajectories. The mean of the conditional distribution $\mathrm{P}\left(\theta_{h}^{\mathrm{f}} \mid \theta_{h}^{\mathrm{c}}\right)$ is then given by

$$
\mu_{\mathrm{f}}^{\text {cond }}=\mu_{\mathrm{f}}+\Sigma_{\mathrm{f}, \mathrm{c}} \Sigma_{\mathrm{c}, \mathrm{c}}^{-1}\left(\mu_{\mathrm{c}}-\theta_{h}^{\mathrm{c}}\right) .
$$

As the inversion of $\Sigma_{\mathrm{c}, \mathrm{c}}$ is the most computationally expensive component of eq. (26), we use at most the last 10 hip angles and heights (sampled at $100 \mathrm{~Hz}$ ) when calculating the conditional mean (compare fig. 3).

\section{Trajectory Planning Quadratic Program Formulation}

To obtain reactive control of the prosthesis swing leg motion inside of the prosthesis' existing Simulink Real-Time environment, we plan future swing trajectories with a fast quadratic program (QP) operating at $100 \mathrm{~Hz}$. The QP includes equality constraints, which ensure the trajectories progress smoothly from the current position to the desired end position, and inequality constraints, which avoid premature ground contact of the toe and heel of the prosthesis. Because in our formulation the QP can only solve for one joint at a time, we first solve for the ankle trajectory assuming the knee trajectory found in the previous time step and then use this updated ankle trajectory to solve for the new knee trajectory.

Figure 4 provides more details of the actions of the trajectory planner algorithm. For example, at a time of about $150 \mathrm{~ms}$ into
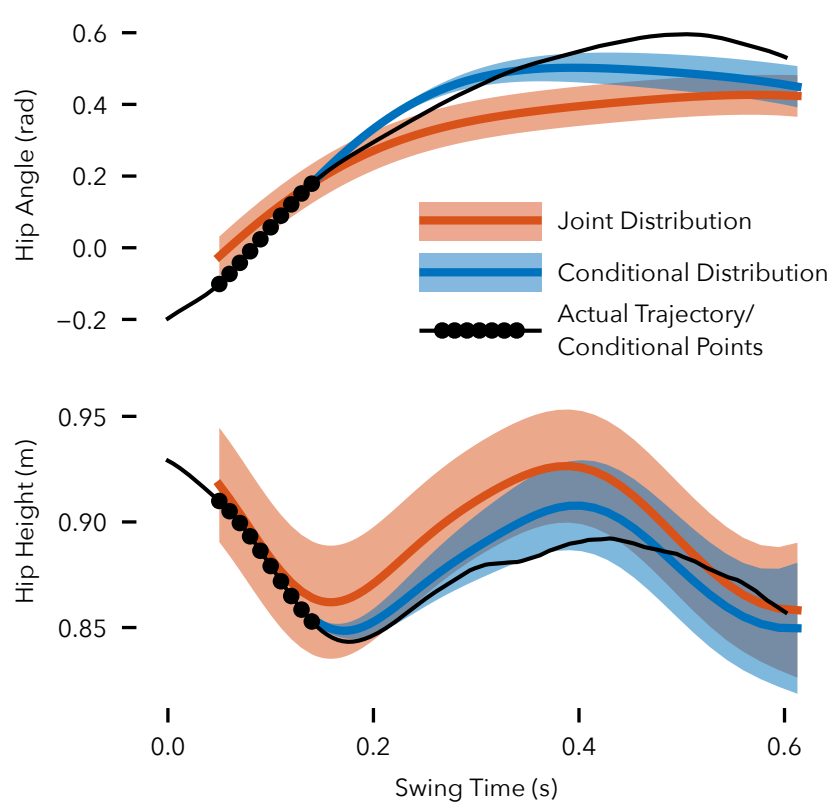

Fig. 3: Example of hip angle and height trajectory predictions $0.15 \mathrm{~s}$ into swing. The prediction algorithm uses the previous 10 measured hip angles and heights (sampled at $100 \mathrm{~Hz}$, black dots) along with the learned joint distributions of hip angles/heights versus time (red) to obtain the conditional distributions of future hip angles/heights (blue). The planning algorithm uses the means of the conditional distributions to generate knee and ankle trajectories. The actual hip height and angle trajectories are shown in black.

the swing phase, the algorithm generates bounds for the knee through inverse kinematics (IK) by solving

$$
\begin{aligned}
\theta_{\mathrm{k}}^{\text {toe bnd }} & =\left\{\theta_{\mathrm{k}}:\left\{p_{\mathrm{OT}}\left(\theta_{\mathrm{h}}, z_{\mathrm{h}}, \theta_{\mathrm{k}}, \theta_{\mathrm{a}}\right)\right\}_{\text {row } 3}=0\right\} \\
\theta_{k}^{\text {heel bnd }} & =\left\{\theta_{\mathrm{k}}:\left\{p_{\mathrm{OL}}\left(\theta_{\mathrm{h}}, z_{\mathrm{h}}, \theta_{\mathrm{k}}, \theta_{\mathrm{a}}\right)\right\}_{\text {row } 3}=0\right\}
\end{aligned}
$$

at a set of sample times spanning the remaining swing trajectory. The QP then uses these bounds to plan the knee trajectory (red trace in fig. 4B). The ankle trajectory is found through a similar procedure. Figures $4 \mathrm{C}$ and $\mathrm{E}$ show the IK solutions at characteristic points in the swing for the knee and ankle respectively, with solutions leading to toe contact shown in purple and solutions leading to heel contact shown in yellow. For each contact point, there are typically two solutions, one lower bound, for which the joint angle cannot cross from above, and one upper bound, for which the joint angle cannot cross from below.

Often, the valid leg configurations span disjointed regions in the configuration space (green and red regions in fig. 4B and D). Therefore, the planner next identifies a valid sequence of regions for the trajectory to traverse in a four step procedure. First, the planner identifies critical points along the predicted trajectory at which any bound activates or deactivates. Second, at each critical point, the planner sorts the bound angles from 


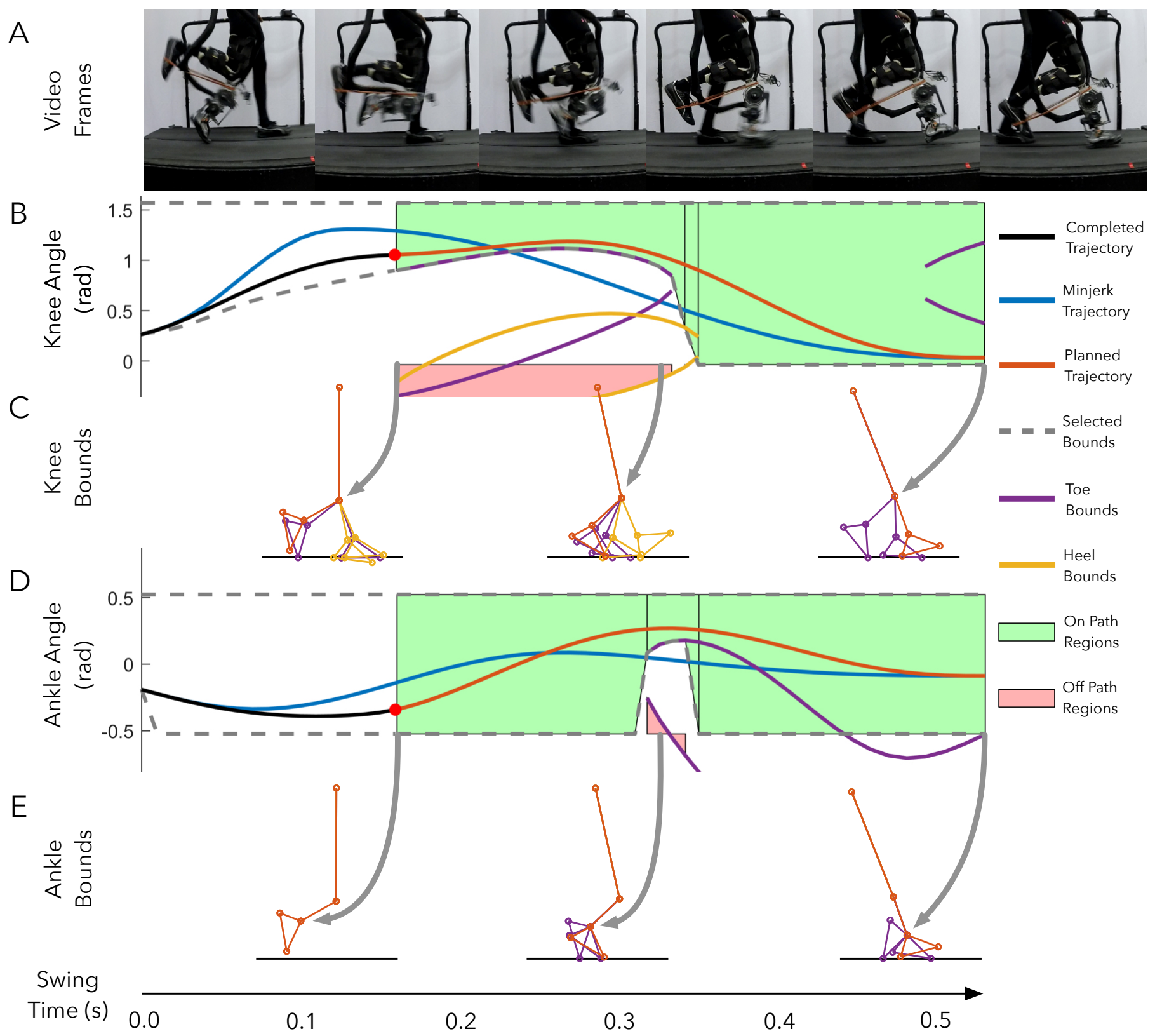

Fig. 4: Planning Algorithm Steps: Panels B and D show the generated knee and ankle trajectories respectively. The planned trajectory (red) lies within the computed bounds (dashed gray). In contrast, standard minimum jerk trajectories (blue) do not respect the bounds, thereby increasing the tripping hazard. Panels $\mathrm{C}$ and $\mathrm{E}$ show examples of inverse kinematics (IK) solutions for toe (purple) and heel (yellow) contact for the knee and ankle joints respectively. We use the IK solutions to generate bounded regions that the planned trajectory can safely traverse. We consider ground contact constraints for only the first half of the remaining swing duration after which we only consider joint angle constraints. We use Dijkstra's algorithm to select regions (green) that allow a path from the start point to the desired final point. Bounded regions that do not lie on the path are shown in red. Panel A shows the corresponding prosthesis motion. 
largest to smallest and iterates through them to define regions between successive upper and lower bounds. Third, the planner defines a graph over the regions with edge weights equal to the average squared angle minus the volume of the child region. This cost favors a sequence of regions that are large and thus safe to travel trough and avoids regions that require excessive joint flexion or extension. Dijkstra's algorithm is then used to find a valid sequence of regions that minimizes this cost [2]. Finally, so that the generated trajectories do not get too close to the identified bounds, a buffer is added to the bounds. This buffer takes the form

$$
\theta_{\text {buf }}=\theta_{\text {buf }}^{0} \sin \left(\pi \frac{t-t_{0}}{t_{f}-t_{0}}\right),
$$

where $\theta_{\text {buf }}^{0}$ is either $5^{\circ}$ for lower bounds or $-5^{\circ}$ upper bounds, $t$ is the future swing time, and $t_{0}$ and $t_{f}$ are the current and final swing times.

After identifying the bounded regions, the planner generates the trajectory for a specific joint by solving a quadratic program. The trajectory of each joint is represented by three, fifth-order polynomial splines,

$$
\begin{aligned}
\theta_{1}(t) & =a_{01}+a_{11} t+\cdots+a_{51} t^{5}=\left[1 t \cdots t^{5}\right] a_{1} \\
T_{0} & \leq t<T_{1} \\
& \vdots \\
\theta_{3}(t) & =a_{03}+a_{13} t+\cdots+a_{53} t^{5}=\left[1 t \cdots t^{5}\right] a_{3} \\
T_{2} & \leq t<T_{F},
\end{aligned}
$$

and solved for by the following QP,

$$
a^{*}=\operatorname{argmin}_{a} \frac{1}{2} a^{T}\left(H_{\theta}+w H_{\ddot{\theta}}\right) a,
$$

where $a=\left[\begin{array}{lll}a_{1}^{T} & a_{2}^{T} & a_{3}^{T}\end{array}\right]^{T}, H_{\theta}$ and $H_{\ddot{\theta}}$ encode quadratic costs on angle and jerk respectively, and $w$ is a weight parameter. The solution is subject to the inequality constraints

$$
\begin{aligned}
& \theta(t) \leq \theta_{\max }(t), \quad \forall t \\
& \theta(t) \geq \theta_{\min }(t), \quad \forall t \\
& \dot{\theta}(t) \leq \dot{\theta}_{\max }, \quad \forall t \\
& \dot{\theta}(t) \geq \dot{\theta}_{\min }, \forall t,
\end{aligned}
$$

which ensure the trajectory lies within the identified bounds and respects velocity limits, and to the equality constraints

$$
\begin{aligned}
\theta\left(T_{0}\right) & =\theta_{0} \\
\dot{\theta}\left(T_{0}\right) & =\dot{\theta}_{0} \\
\ddot{\theta}\left(T_{0}\right) & =\ddot{\theta}_{0} \\
\theta\left(T_{F}\right) & =\theta_{F} \\
\dot{\theta}\left(T_{F}\right) & =0 \\
\ddot{\theta}\left(T_{F}\right) & =0 \\
\theta_{1}\left(T_{1}\right) & =\theta_{2}\left(T_{1}\right) \\
\dot{\theta}_{1}\left(T_{1}\right) & =\dot{\theta}_{2}\left(T_{1}\right) \\
\ddot{\theta}_{1}\left(T_{1}\right) & =\ddot{\theta}_{2}\left(T_{1}\right)
\end{aligned}
$$

which ensure the trajectory starts at the current and terminates at the desired positions, velocities, and accelerations and that the splines join together smoothly. If the QP fails to find a trajectory that can satisfy the constraints, the last found valid trajectory is reused for the next time step. In addition, at the first iteration, the ankle trajectory planner uses the output of the minimum jerk trajectory planner to solve the inverse kinematics for the bounds.

\section{Experimental Procedure}

We tested the ability of the proposed trip avoidance control to reduce the incidence and severity of trips while walking with the powered transfemoral prosthesis shown in fig. 1 [25]. To evaluate the performance of the system, an able-bodied user walked with the prosthesis while attempting to elicit trips by lowering the hip in swing. During the stance phase, the prosthesis randomly decided to either use the proposed swing control or to use standard minimum jerk trajectories that do not consider the tripping hazard. The user was not aware of which controller would be used in the upcoming swing. The user completed a total of ten one minute walking trials.

We examined several outcomes for evaluating control performance. First, we examined the distribution of knee angles at the beginning of stance. Large knee angles at the beginning of stance indicate premature landing due to toe-strike instead of heel strike. Ideally, the landing angle is close to the desired final angle of 2 degrees. Second, we checked the integral of the ground reaction force during swing. If this quantity is large, it indicates scuffing of the toe on the ground. Finally, we examined the relationship between the hip and toe heights during swing. If our controller is working as intended, the toe height during swing should have a decreased sensitivity to the hip height.

\section{Results}

Figure 5 shows the knee and ankle swing trajectories generated by the proposed control (blue) and by a standard jerk minimization control (red) during normal walking and trip elicitation. During undisturbed walking, the trajectories produced by both control strategies are similar. However, the proposed control strategy has a tendency to keep the knee flexed for longer and then extends it faster towards the end of swing. In addition, in a few steps, the proposed controller flexed the ankle significantly more than did the standard minimum jerk control. These trends are exaggerated during trip elicitation. There are more knee trajectories in which the knee stays flexed for longer, thereby creating more ground clearance. In addition, the ankle flexes earlier, which will help to create more foot clearance when the hip is suddenly lowered in early swing.

We used video and audio recordings of the trials, as well as data from the prosthesis, to manually classify trips as those swing trajectories that end with toe strike or during which the foot scuffed on the ground. We find that over the ten minutes of walking, the minimum jerk control produced 109 trips while the proposed approach produced 35 trips, reducing the trip rate by $68 \%$. 


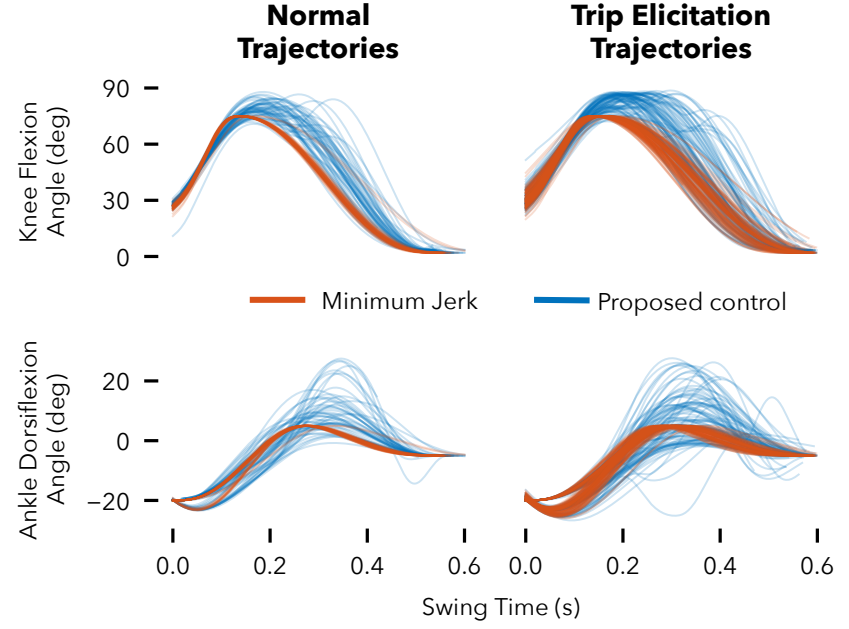

Fig. 5: Knee and ankle trajectories produced during normal walking and while eliciting trips. To avoid tripping during trip elicitation trials, trajectories generated by the proposed approach often flex the knee to a greater degree and for longer before quickly extending at the end of swing. At the ankle joint we see overall greater variability in the generated trajectories during the trip elicitation condition versus normal walking.

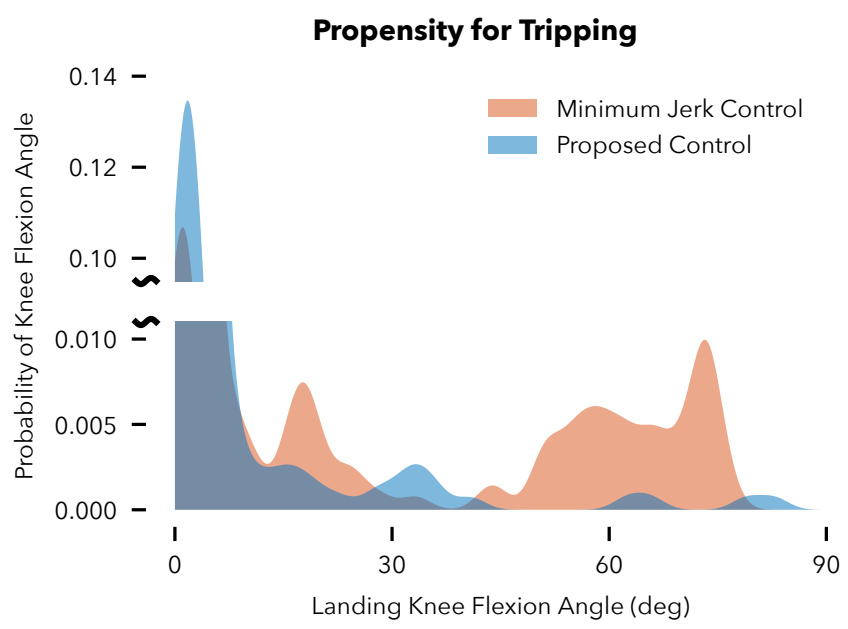

Fig. 6: Kernel density estimate of the probability of various landing knee flexion angles with the proposed swing control (blue) and standard min-jerk swing control (red). Large landing knee angles indicate premature toe contact during swing.

To further examine the performance of the two control strategies, we used kernel density estimates of the landing knee flexion angle, a measure of the propensity for tripping, and integrated ground reaction force (GRF) during swing, a measure of the propensity for foot scuffing. Figure 6 shows the distributions of the landing angle of the prosthesis at the end of swing for the proposed swing control (blue) and for the standard minimum jerk swing control (red) during the trip elicitation condition. We observe the minimum jerk control

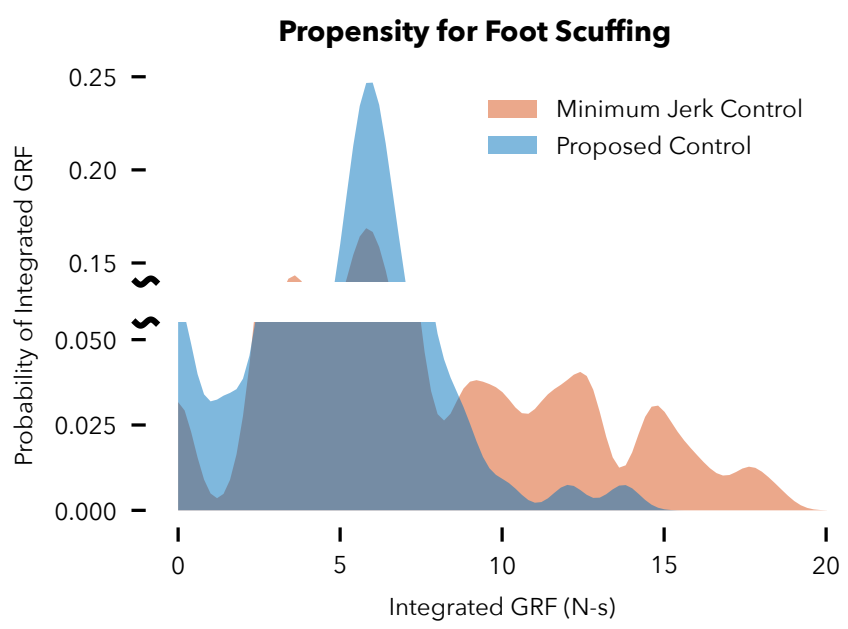

Fig. 7: Kernel density estimate of the probability of various integrated ground reaction force values for the proposed swing control (blue) and standard min-jerk swing control (red). Large integrated GRF during the swing phase is indicative of the toe scuffing on the ground.

is much more likely to generate a swing trajectory that ends prematurely with a large knee flexion angle, which is indicative of toe contact instead of heel contact at the end of swing. The distributions of the integrated GRFs suggests the minimum jerk control produced a larger percentage of swings with high ground reaction forces than the proposed control, indicating an increased frequency and severity of toe scuffing during swing (fig. 7).

We can also ask the question, "For steps during which the prosthesis used trajectories generated by the proposed control, would the user have tripped had the prosthesis used a minimum jerk trajectory?" To answer this question, we can use the kinematics model shown in fig. 1 along with ground truth hip height and hip angle data captured via a motion capture system, to estimate the location of the toe had the knee and ankle perfectly followed the desired trajectories produced by each control scheme. This analysis predicts that the prosthesis would have tripped or scuffed the toe on the ground during $22 \%$ of the steps if we had used the minimum jerk trajectory. In contrast, it predicts a trip or scuff rate of $5 \%$ had we perfectly followed the trajectories generated by the proposed control.

Finally, fig. 8 shows the relationship between the average toe and hip heights during swing for both control schemes. The toe height of the prosthesis, when controlled by the proposed control, is less sensitive to decreases in the hip height than it is when using the standard minimum jerk control.

\section{Discussion}

We presented initial work toward a real-time reactive control of powered prostheses to help amputees avoid tripping in the swing phase of gait. At any time during swing, the proposed control uses a laser range finder and an inertial 


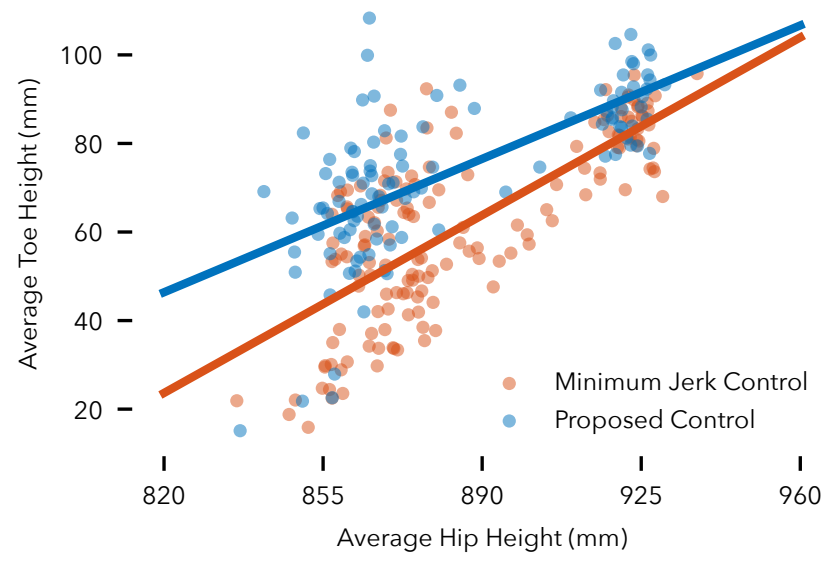

Fig. 8: Average toe height vs average hip height for the proposed swing control (blue) and standard min-jerk swing control (red). The toe height during swing is less sensitive to the hip height when using the proposed swing control than when using the min-jerk swing control.

measurement unit to estimate the current pose of the prosthetic leg, predict the future hip angle and height based on trained Gaussian process models, and plan new knee and ankle joint trajectories that ensure neither the toe nor heel contact the ground prematurely. Our results indicate the proposed control approach can substantially reduce the incidence of trips and reduce the severity and frequency of toe scuffing.

To the best of our knowledge, this work is the first demonstration of lower limb prosthetic control that integrates perception feedback in real-time and that proactively ameliorates the falling hazard amputees face. Previous research in this area has largely focused on detecting stumbles after they have occurred. For example, Lawson et al. [11] and Shirota et al. [22] have proposed classifiers that can detect trips during swing and predict whether a lower or raising strategy should be used in response. Similarly, Zhang et al. [26] have proposed a method that can detect stumbles and classify them as trips during swing or slips during stance. However, these previous studies have not proposed concrete control actions to preempt stumbles or to properly react in the event that a stumble is detected. Our results motivate further research into such proactive and reactive approaches, closing the perception-action loop for improving gait robustness with robotic prostheses.

Several avenues for future work exist. First, in our current study, only one able-bodied user tested the proposed control. Further experiments with amputee subjects are needed to verify the system provides benefits to this population. For instance, amputees accustomed to walking with passive prostheses show significantly altered hip kinematics [9], which could affect the control behavior. However, the proposed control should be able to properly adapt to these behavior differences, as the Gaussian process models are trained for specific users. Second, although trips during swing are one of the most common failure modes we encounter with our powered prostheses, these events are still rare and many hours of normal walking are required to observe a sufficient number of trips to compare controllers. As a result, we actively induced trips by sudden drops in hip height during swing, which does not exactly reflect the situations in which trips occur. Specifically, trips can happen due to subtle changes in leg kinematics, and it remains to be seen in experiments if our approach can avoid trips in these more subtle situations.

At the implementation level, there is also room for further exploration. To keep the computational costs low and to facilitate implementation in Simulink Real-time, we used quadratic programs that iterate between finding solutions for the ankle and knee joints. While this iterative approach is fast when compared to trajectory optimization methods that deal with multiple joints simultaneously, the iterations occasionally get stuck when the planner for one joint trajectory cannot find a solution based on the assumed fixed trajectory of the other joint. Moreover, if a solution cannot be found, the current approach simply reuses the last identified trajectory, rather than moving the trajectory to be more safe, even if it cannot fully satisfy the bounds. It seems worthwhile to investigate whether non-convex trajectory optimization methods such as CHOMP [20], in which the bounds are represented as soft rather than hard constraints, can help solve for the knee and ankle trajectories simultaneously without sacrificing computational speed.

In addition, several technical simplifications can be considered to bring this technology closer to commercialization. We used an accurate and relatively expensive laser distance sensor, eyeing future research toward obstacle scanning and avoidance capabilities. However, for simple ground plane avoidance, inexpensive infrared distance sensors such as those used by Scandaroli et al. [21] are likely sufficient. It may also be possible to simplify the trajectory planning phase by, for example, forgoing formal guarantees on satisfying bounds and instead relying on heuristics to increase knee and ankle flexion and adjust timing in response to decreased hip height during swing.

Our immediate goal, however, is to generalize the presented approach to incorporating perception in control beyond the avoidance of flat ground. We are currently investigating how to extend the approach to plan trajectories around obstacles that are scanned by the laser range finder. Previous studies such as Mohagheghi et al. [17] with able-bodied subjects have shown that vision plays a crucial role in both planning and control of the lower limb motion over obstacles. We also envision using the approach to target objects instead of avoiding them. For example, a prosthetic leg could scan, recognize, and target secure footholds and stair treads, or provide enhanced sports capabilities by targeting and kicking a ball.

\section{REFERENCES}

[1] Brian DO Anderson and John B Moore. Optimal filtering. Englewood Cliffs, 21:22-95, 1979.

[2] Edsger W Dijkstra. A note on two problems in connexion with graphs. Numerische mathematik, 1(1):269-271, 1959.

[3] Lin Du, Fan Zhang, Ming Liu, and He Huang. Toward design of an environment-aware adaptive locomotion-mode- 
recognition system. IEEE Transactions on Biomedical Engineering, 59(10):2716-2725, 2012.

[4] Ran Duan, Shuangyue Yu, Guang Yue, Richard Foulds, Chen Feng, Yingli Tian, and Hao Su. Real-time robust $3 \mathrm{~d}$ plane extraction for wearable robot perception and control. In 2018 Design of Medical Devices Conference, pages V001T06A003-V001T06A003. American Society of Mechanical Engineers, 2018.

[5] E Foxlin. Pedestrian tracking with shoe-mounted inertial sensors. IEEE Computer Graphics and Applications, 25 (6):38-46, 2005.

[6] Christiane Gauthier-Gagnon, Marie-Claude Grisé, and Diane Potvin. Enabling factors related to prosthetic use by people with transtibial and transfemoral amputation. Archives of physical medicine and rehabilitation, 80(6): 706-713, 1999.

[7] Nachi Gupta and Raphael Hauser. Kalman filtering with equality and inequality state constraints. arXiv preprint arXiv:0709.2791, 2007.

[8] Yoshiaki Hayashi and Kazuo Kiguchi. A lower-limb power-assist robot with perception-assist. In 2011 IEEE International Conference on Rehabilitation Robotics, pages 1-6. IEEE, 2011.

[9] Sonja MHJ Jaegers, J Hans Arendzen, and Henry J de Jongh. Prosthetic gait of unilateral transfemoral amputees: a kinematic study. Archives of physical medicine and rehabilitation, 76(8):736-743, 1995.

[10] Nili Eliana Krausz, Tommaso Lenzi, and Levi J Hargrove. Depth sensing for improved control of lower limb prostheses. IEEE Transactions on Biomedical Engineering, 62 (11):2576-2587, 2015.

[11] Brian E Lawson, H Atakan Varol, Frank Sup, and Michael Goldfarb. Stumble detection and classification for an intelligent transfemoral prosthesis. In 2010 Annual International Conference of the IEEE Engineering in Medicine and Biology, pages 511-514. IEEE, 2010.

[12] Tommaso Lenzi, Levi Hargrove, and Jonathon Sensinger. Speed-adaptation mechanism: Robotic prostheses can actively regulate joint torque. IEEE Robotics \& Automation Magazine, 21(4):94-107, 2014.

[13] Ming Liu, Ding Wang, and He Helen Huang. Development of an environment-aware locomotion mode recognition system for powered lower limb prostheses. IEEE Transactions on Neural Systems and Rehabilitation Engineering, 24(4): 434-443, 2016.

[14] Sebastian Madgwick. An efficient orientation filter for inertial and inertial/magnetic sensor arrays. Technical report, University of Bristol (UK), 2010.

[15] Yerzhan Massalin, Madina Abdrakhmanova, and Huseyin Atakan Varol. User-independent intent recognition for lower-limb prostheses using depth sensing. IEEE Transactions on Biomedical Engineering, 2017.

[16] William C Miller, Mark Speechley, and Barry Deathe. The prevalence and risk factors of falling and fear of falling among lower extremity amputees. Archives of physical medicine and rehabilitation, 82(8):1031-1037, 2001.

[17] Amir A Mohagheghi, Renato Moraes, and Aftab E Patla. The effects of distant and on-line visual information on the control of approach phase and step over an obstacle during locomotion. Experimental Brain Research, 155(4): 459-468, 2004.

[18] David Quintero, Dario J Villarreal, and Robert D Gregg. Preliminary experiments with a unified controller for a powered knee-ankle prosthetic leg across walking speeds. In Proceedings of the... IEEE/RSJ International Conference on Intelligent Robots and Systems. IEEE/RSJ International Conference on Intelligent Robots and Systems, volume 2016, page 5427. NIH Public Access, 2016.

[19] Carl Edward Rasmussen. Gaussian processes in machine learning. In Advanced lectures on machine learning, pages 63-71. Springer, 2004.

[20] Nathan Ratliff, Matt Zucker, J Andrew Bagnell, and Siddhartha Srinivasa. Chomp: Gradient optimization techniques for efficient motion planning. In Robotics and Automation, 2009. ICRA'09. IEEE International Conference on, pages 489-494. IEEE, 2009.

[21] Glauco Garcia Scandaroli, Geovany Araújo Borges, João Yoshiyuki Ishihara, Marco Henrique Terra, Adson Ferreira da Rocha, and Francisco Assis de Oliveira Nascimento. Estimation of foot orientation with respect to ground for an above knee robotic prosthesis. In Intelligent robots and systems, 2009. IROS 2009. IEEE/RSJ international conference on, pages 1112-1117. IEEE, 2009.

[22] Camila Shirota, Ann M Simon, and Todd A Kuiken. Recovery strategy identification throughout swing phase using kinematic data from the tripped leg. In 2014 36th Annual International Conference of the IEEE Engineering in Medicine and Biology Society, pages 6199-6202. IEEE, 2014.

[23] Edward Snelson and Zoubin Ghahramani. Local and global sparse gaussian process approximations. In Artificial Intelligence and Statistics, pages 524-531, 2007.

[24] Frank Sup, Huseyin Atakan Varol, Jason Mitchell, Thomas J Withrow, and Michael Goldfarb. Preliminary evaluations of a self-contained anthropomorphic transfemoral prosthesis. IEEE/ASME Transactions on mechatronics, 14(6):667-676, 2009.

[25] Nitish Thatte. Design and Evaluation of Robust Control Methods for Robotic Transfemoral Prostheses. PhD thesis, Pittsburgh, PA, May 2019.

[26] Fan Zhang, Susan E D’Andrea, Michael J Nunnery, Steven M Kay, and He Huang. Towards design of a stumble detection system for artificial legs. IEEE Transactions on Neural Systems and Rehabilitation Engineering, 19(5):567577, 2011.

[27] Fan Zhang, Zheng Fang, Ming Liu, and He Huang. Preliminary design of a terrain recognition system. In Engineering in medicine and biology society, EMBC, 2011 annual international conference of the IEEE, pages 54525455. IEEE, 2011. 